\title{
Deformation and stress distribution of the human foot after plantar ligaments release: A cadaveric study and finite element analysis
}

\author{
LIANG Jun ${ }^{1}$, YANG YunFeng ${ }^{2}$, YU GuangRong ${ }^{2}$, NIU WenXin ${ }^{3} \&$ WANG YuBin ${ }^{1 *}$ \\ ${ }^{1}$ Sports Medicine Department of Shanghai East Hospital, Tongji University, Shanghai 200120, China, \\ ${ }^{2}$ Orthopaedic Department of Tongji Hospital, Tongji University, Shanghai 200065, China; \\ ${ }^{3}$ School of Biological Science and Medical Engineering, Beihang University, Beijing 100191, China
}

Received July 24, 2010; accepted November 13, 2010

\begin{abstract}
The majority of foot deformities are related to arch collapse or instability, especially the longitudinal arch. Although the relationship between the plantar fascia and arch height has been previously investigated, the stress distribution remains unclear. The aim of this study was to explore the role of the plantar ligaments in foot arch biomechanics. We constructed a geometrical detailed three-dimensional (3-D) finite element (FE) model of the human foot and ankle from computer tomography images. The model comprised the majority of joints in the foot as well as bone segments, major ligaments, and plantar soft tissue. Release of the plantar fascia and other ligaments was simulated to evaluate the corresponding biomechanical effects on load distribution of the bony and ligamentous structures. These intrinsic ligaments of the foot arch were sectioned to simulate different pathologic situations of injury to the plantar ligaments, and to explore bone segment displacement and stress distribution. The validity of the 3-D FE model was verified by comparing results with experimentally measured data via the displacement and von Mise stress of each bone segment. Plantar fascia release decreased arch height, but did not cause total collapse of the foot arch. The longitudinal foot arch was lost when all the four major plantar ligaments were sectioned simultaneously. Plantar fascia release was compromised by increased strain applied to the plantar ligaments and intensified stress in the midfoot and metatarsal bones. Load redistribution among the centralized metatarsal bones and focal stress relief at the calcaneal insertion were predicted. The 3-D FE model indicated that plantar fascia release may provide relief of focal stress and associated heel pain. However, these operative procedures may pose a risk to arch stability and clinically may produce dorsolateral midfoot pain. The initial strategy for treating plantar fasciitis should be non-operative.
\end{abstract}

biomechanics, finite element analysis, foot arch, ligament, stress

Citation: Liang J, Yang Y F, Yu G R, et al. Deformation and stress distribution of the human foot after plantar ligaments release: A cadaveric study and finite element analysis. Sci China Life Sci, 2011, 54: 267-271, doi: 10.1007/s11427-011-4139-0

The plantar fascia, or plantar aponeurosis, is the investing fascial layer of the plantar aspect of the foot, and forms part of the retinacular system, consisting of a network of connective and adipose tissues that supports and protects underlying vital structures of the body. The anatomy of the plantar fascia has been well described by Sarrafian [1] and

*Corresponding author (email: wybdf@yahoo.com.cn) others [2-5]. Partial or total plantar fascia release may relieve metatarsal and calcaneal stresses and painful heel syndromes of plantar fasciitis. However, reduction of plantar fascia stiffness may have a significant impact on arch stability, resulting in a more deformable longitudinal arch.

Cadaveric studies have been performed to examine biomechanical consequences of release of the plantar ligaments. Huang [6] reported that the average vertical displacements 
between the talar neck and a supporting platform were 7.3 and $8.4 \mathrm{~mm}$, respectively, in 12 cadaveric feet with intact plantar fascia and fasciotomy under a load of $690 \mathrm{~N}$. In a biomechanical cadaver study, Kitaoka et al. [7,8] noted that high tensile loads were required for failure of the plantar fascia. Further, in that study the majority of failures or ruptures during testing occurred at the plantar fascia origin from the os calcis, the most common site of clinical plantar fascial rupture and symptoms of plantar fasciitis. Daly et al. [9] also reported flattening of the arch in 16 feet from 13 patients who underwent plantar fasciotomy for intractable plantar fasciitis. Because of the intrinsic structural and material variability of cadaver specimens and experimental limitations, systematic evaluation of the stress distribution and movement of the bony segments of the ankle-foot complex after plantar fascia release are difficult. Further, although computational approaches have been used to acquire these biomechanical parameters, these mathematic models were not verified with cadaveric experiments under similar pathologic situations $[10,11]$.

The aim of the present study was to establish a detailed finite element (FE) model of a normal human adult foot and to analyze foot arch deformation and stress distribution after plantar fascia release based on FE model prediction and cadaveric experiment verification.

\section{Materials and methods}

The geometry of the finite element model was obtained from a 27-year-old male (height $175 \mathrm{~cm}$, weight $70 \mathrm{~kg}$ ) without any foot pathology. A series of coronal computer tomography (CT) images of $2 \mathrm{~mm}$ intervals in a neutral unloaded position were segmented to obtain the boundaries of skeleton and plantar soft tissue. The contours of the bone and soft tissue were determined by an automatic contouring program, and were used to generate the solid models by a CAD program (AutoCADR14.0, Auto Computer Aided Design, Autodesk, Inc., USA). The four-node tetrahedral models were created and analyzed using a CAE program (ANSYS9.0, ANSYS, Inc., Canonsburg, Pennsylvania, USA). The articulations and ligament structures of the foot were created with the Link 10 and Link 12 models, respectively, and the Shell 93 model was used to construct the plantar soft tissues (Figure 1). The material properties were assumed to be linearly elastic. A detailed left foot model was built, which consisted of 170426 nodes, including articular cartilage, ligaments, and plantar soft tissue. Static loading of $700 \mathrm{~N}$ was employed axially through the distal tibia to simulate the one foot standing with the heel of the model fixed and the other part of the plantar soft tissue elements restrained in the vertical axis and free in the transverse plane. All nodes on the upper cross-sectional area of the distal tibia were restrained in the transverse plane but free in the coronal axis. Next, a rigid plane under the foot plantar soft tissues was established to simulate the ground. The reaction of each bone segment of the foot arch was recorded and analyzed.

Seven fresh adult cadaveric feet (the $1 / 3$ part of the shanks were attached) were tested. The skin and muscles above the ankle joint were detached, while the ligaments of the ankle were kept intact at the same time (Figure 2). The four major bone segments and stabilizers of the foot arch (plantar fascia, spring ligament, long, and short plantar ligament) were identified and marked before experimentation. A $700 \mathrm{~N}$ axial load from the proximal tibia was applied by MTS (Material Test System, MTS Systems Corporation, USA) in a $100 \mathrm{~N}$ gradient. Simulation of ligament injury was performed using different combinations and sequences of ligament release. Displacement of the major bones was collected in gray level images by two digital cameras and recorded by a computer. Displacement of the bone segments was calculated by Digital Speckle Correlated Methods and compared with the FE model results for verification. The FE model angular displacements of the major tarsal bones were also analyzed based on the lines that passed through the middle points of the articulations including the subtalar joint, talonavicular joint, chopart joint, Lisfranc joint, and metatarsophalangeal joints.
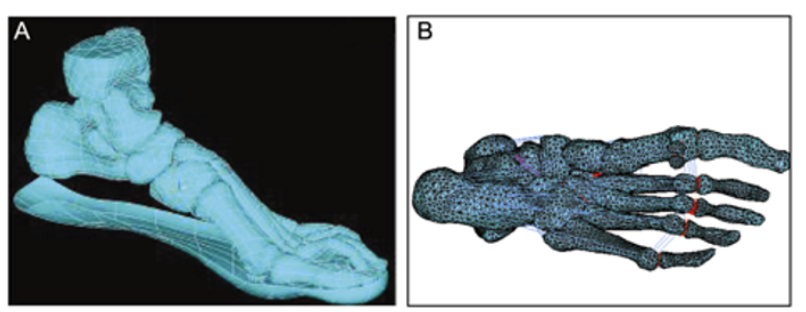

Figure 1 The solid model of the foot and plantar soft tissue (A). The plantar ligaments and intrinsic connections were built with different units (B).

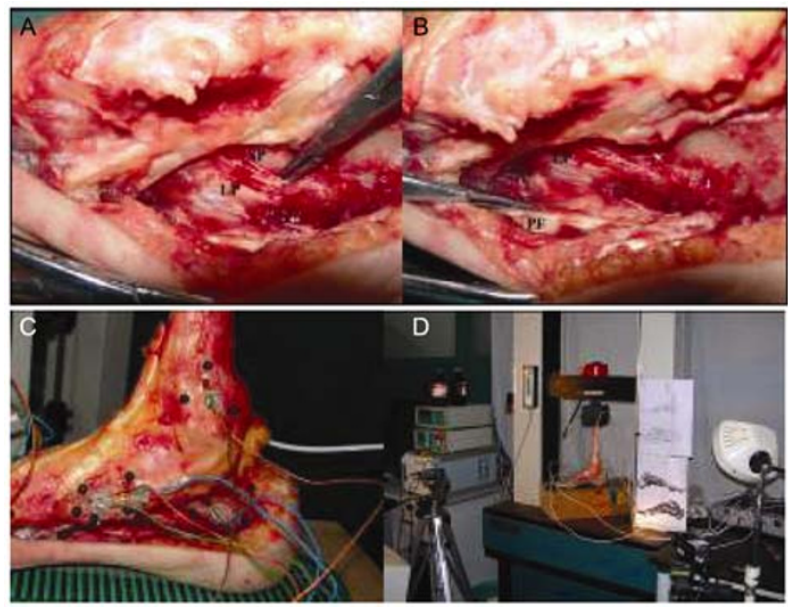

Figure 2 Cadaveric feet were sectioned to expose the plantar fascia (PF) and other major ligaments (LP, long plantar ligament; SP, short plantar ligament) and loaded with MTS to $700 \mathrm{~N}$ axially. Displacements of the marked bone segments were recorded with two digital cameras. The plantar pressure was measured by an F-Scan Mobile system. 


\section{Results}

All the marked bone segments moved downward in the sagittal plane under axial load in the intact situation. The calcaneus showed plantarflexion, while the other tarsal and metatarsal bones exhibited dorsiflexion, which led to longitudinal and transversal arch flattening in the model. The FE model and the cadaveric feet showed the same pattern when a $700 \mathrm{~N}$ load was applied to the distal part of the tibial in the intact situation, except for the calcaneus and the fifth metatarsal. When all the four plantar ligaments were sectioned, all the bone segments appeared to displace in all three global planes, i.e., dorsiflexion in the sagittal plane, abduction in the transverse plane, and external rotation in the coronal plane (Figure 3). The FE model and the cadaveric experiment showed a similar pattern (Figure 4). The rotation changes between intact, plantar fascia released, and all four major ligaments released of the FE model are shown in Figures 5-7, respectively.

The bone segments rotated in the three planes and

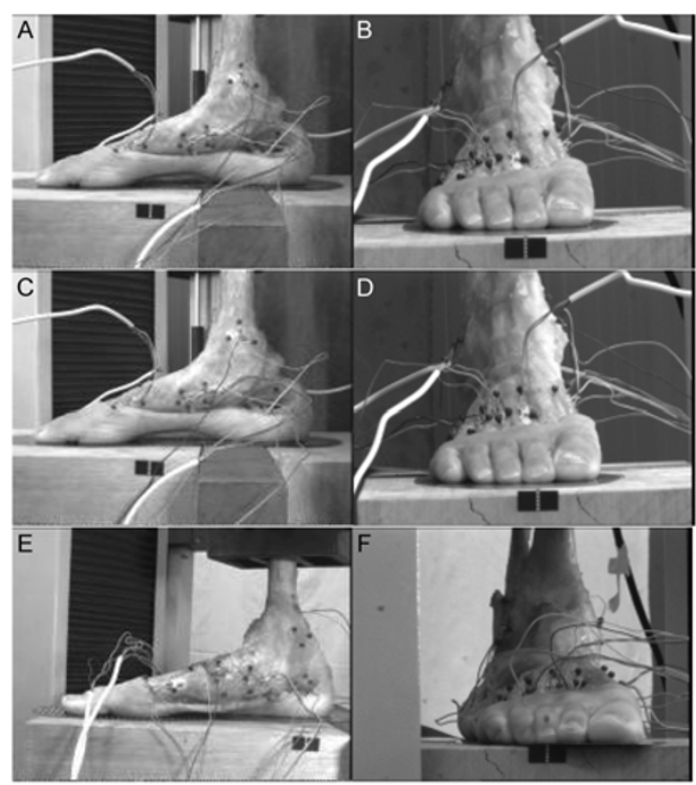

Figure 3 Vertical displacement of the cadaveric foot in sagittal and coronal planes captured by two digital cameras under $700 \mathrm{~N}$ loading before (A and $\mathrm{B}$ ) and after $(\mathrm{C}$ and $\mathrm{D})$ the plantar fascia was released and the four plantar ligaments were sectioned $(\mathrm{E}$ and $\mathrm{F})$.

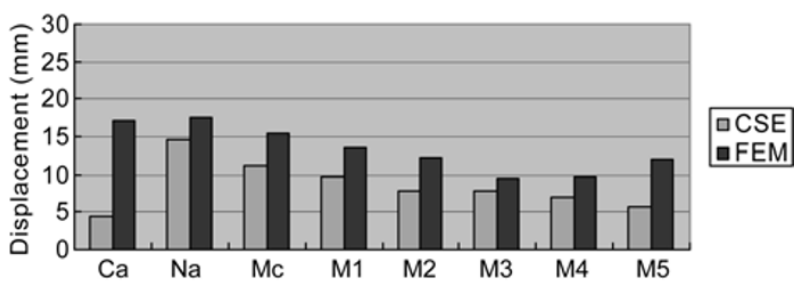

Figure 4 The vertical displacement of the bone segments under $700 \mathrm{~N}$ axial loading in the intact finite element model (FEM) and cadaveric foot specimen experiment (CSE) showed the same pattern, except for the calcaneus and the fifth metatarsal.

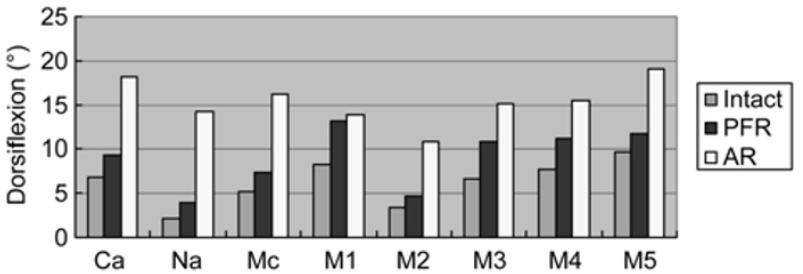

Figure 5 Rotation of the bone segments in the sagittal plane under $700 \mathrm{~N}$ axial loading following release of the intact, plantar fascia (PFR) and the four major plantar ligaments (AR).

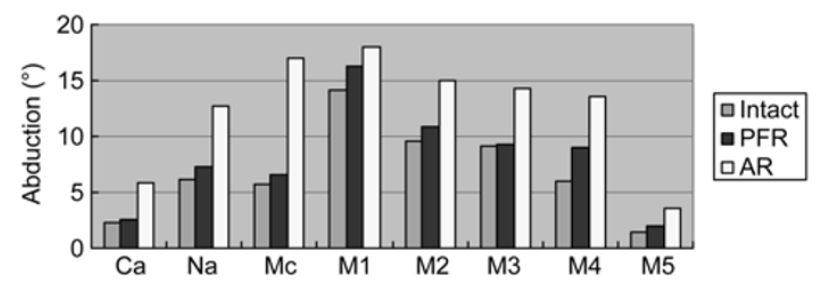

Figure 6 Rotation of the bone segments in the transverse plane under $700 \mathrm{~N}$ axial loading following release of the intact, plantar fascia (PFR) and the four major plantar ligaments (AR).

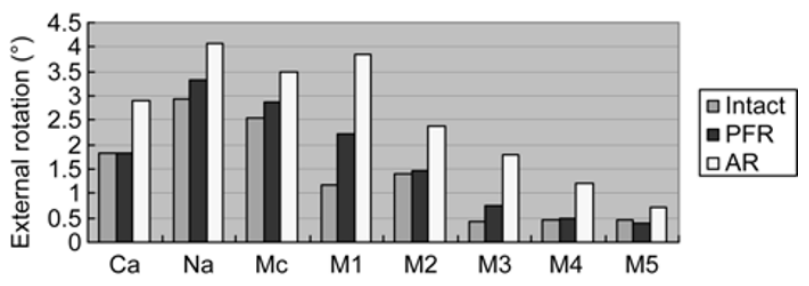

Figure 7 Rotation of the bone segments in the coronal plane under $700 \mathrm{~N}$ axial loading following release of the intact, plantar fascia (PFR) and the four major plantar ligaments (AR).

showed the same pattern, except for the calcaneus and the fifth metatarsal in the sagittal plane. Following flattening of the longitudinal arch the foot bones showed dorsiflexion in the sagittal plane, abduction in the transversal plane, and external rotation in the coronal plane. The degrees of the rotation changed when the plantar ligaments were released, and peaked as the four major ligaments were all sectioned (Figures 5-7). The model decreased markedly by vision in all phases after the plantar fascia was released, but moved only slightly in the other condition. The arch of the model showed the greatest decrease when all the ligaments were sectioned, and the largest von Mise stress was found in the lateral mid-foot region (Figure 8). The plantar pressure showed the same pattern (Figure 9). The abnormal redistribution of the fore foot stress may lead to overuse injury of the metatarsal region. When the total stability was set at $100 \%$, the calculated contribution of the plantar fascia to the stability of the foot arch was $34.53 \%, 22.46 \%$, and $12.63 \%$ in sagittal, transversal, and coronal planes, respectively.

\section{Discussion}

Computer models have several benefits over other types of 

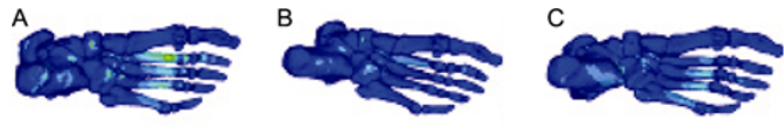

Figure 8 The von Mise stress distribution of the FE model under $700 \mathrm{~N}$ vertical load before (A) and after (B) release of the plantar fascia and sectioning of the four plantar ligaments $(\mathrm{C})$

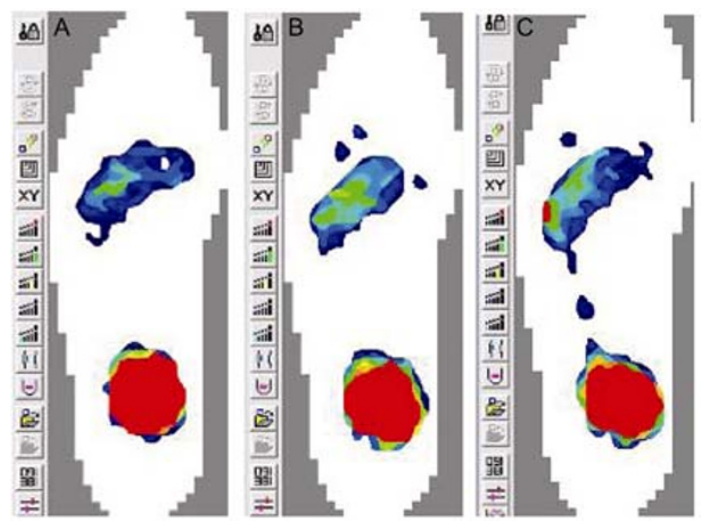

Figure 9 Plantar pressure distribution of the cadaveric foot under $700 \mathrm{~N}$ vertical load before (A) and after (B) release of the plantar fascia and sectioning of the four plantar ligaments (C). The red color represents high pressure.

models [12]. For example, an infinite number of computer models can be developed and tested for different conditions, and the primary characteristic of the model is maintained after exhaustive testing. Computer models also provide information that cannot be easily obtained using other types of models, such as load distributions within soft tissues, internal stresses, joint reaction forces, and muscle force analysis [13]. Once an accurate computer model has been developed and validated appropriately, simulations can be performed quickly and at low cost in diverse situations including injury, surgery, dynamic motion simulation, and graphical animation of the experiment. Further, these models help to determine the underlying mechanisms. The capability of the FE model to predict the internal stress within the bony and soft tissue structures makes it a valuable tool in the study of ankle-foot biomechanics.

Computational analysis of foot biomechanics provides an overall stress distribution of the foot. It is also more economical than in vitro cadaver experiments. Nevertheless, computer models require a detailed representation of foot geometry and joint characteristics, together with realistic loading conditions, to depict the internal stress and strain distributions of the foot complex. FE models have been widely used to quantify the biomechanical role of the plantar fascia in load bearing, and vertical displacement of the foot was found to increase with fasciotomy [10,11]. 3-D geometrical detailed FE models have also been developed [14-19], although those studies did not quantify the biomechanical role of the plantar fascia to the tarsal and metatarsal bone simultaneously, and did not examine the one-foot standing posture, which is important to support the body weight in walking. Based on both anatomy and computer software, we established a detailed FE model of a normal adult left foot that included the bone segments, articulations, foot intrinsic ligaments, and plantar soft tissue. The elements used to establish the foot joints, ligaments, and plantar tissue were distinct from those previously reported, which could not be used to analyze significant displacement of the foot arch.

Plantar fasciitis is an inflammatory condition of the plantar fascia, the strong, fibrous band that originates from the calcaneus and extends distal to the phalanges. Plantar fasciitis affects approximately two million people in the United States per year, and accounts for approximately $10 \%$ of runner-related injuries [3]. Plantar fascia release for chronic plantar fasciitis has provided excellent pain relief and rapid return to activity with only few reported complications, although later outcomes were not examined, some patients suffered a flatten foot and occurrence of lateral pain of the mid-foot. However, cadaveric studies have identified potential postoperative problems including weakness of the medial longitudinal arch and pain in the lateral midfoot $[5,20]$. In the present study, sectioning of the plantar fascia resulted in pronounced reduction of arch height during load bearing, but did not result in total collapse of the foot arch even with additional dissection of the long plantar ligament. Similar results were previously reported in a cadaver study [7]. In our study, we simulated the contribution of the plantar fascia in supporting the longitudinal foot arch, which comprised $1 / 3$ of the function of plantar ligaments in sagittal plane and helped to restrict abduction and external rotation of the bone structure. Plantar fasciotomy should be the last option in clinical therapy, following use of conservative methods.

To simplify the FE model analysis, homogeneous and linearly elastic material properties were assigned to the bony and ligamentous structures, while the ligaments within the toes and other connective tissues such as the joint capsules were not considered. The current FE model did not account for the surface interactions between bony, ligamentous, and muscles structures. The structural simplification of the FE model resulted in a reduction of joint stability of the foot arch structures and an increase in prediction of joint and arch deformation. As we used linear truss elements to approximate the nonlinear profile of the plantar fascia structure, assumed a linear material property, and neglected the interface between the plantar fascia and surrounding tissue, the predicted plantar fascia strain in this study was likely underestimated.

\section{Conclusion}

All the four plantar ligaments play an important role in stabilizing the normal foot arch, particularly the plantar fascia. 
The medial longitudinal foot arch collapses and elongates markedly following forefoot abduction and hind foot valgus because of release of the four plantar ligaments without the function of the tendons and extrinsic stabilizer. We propose a validated 3-D foot model that can be modified to simulate multiple foot conditions, and that is useful for examining stress distributions inside the foot, designing footwear, investigating the biomechanical behavior of the foot subjected to different injuries, and operation design.

This work was supported by the National Natural Science Foundation of China (Grant No. 30801163).

1 Sarrafian S K. Functional characteristics of the foot and plantar aponeurosis under tibiotalar loading. Foot Ankle, 1987, 8: 4-18

2 Murphy G A, Pneumaticos S G, Kamaric E, et al. Biomechanical consequences of sequential plantar fascia release. Foot Ankle Int, 1998, 19: 149-152

3 Riddle D L, Pulisic M, Sparrow K. Impact of demographic and impairment-related variables on disability associated with plantar fasciitis. Foot Ankle Int, 2004, 25: 311-317

4 Snider M P, Clancy W G, McBeath A A. Plantar fascia release for chronic plantar fasciitis in runners. Am J Sports Med, 1983, 11: 215-219

5 Taniguchi A, Tanaka Y, Takakura Y, et al. Anatomy of the spring ligament. J Bone Joint Surg Am, 2003, 85: 2174-2178

6 Huang C K, Kitaoka H B, An K N, et al. Biomechanical evaluation of longitudinal arch stability. Foot Ankle, 1993, 14: 353-357

7 Kitaoka H B, Luo Z P, An K N. Analysis of longitudinal arch supports in stabilizing the arch of the foot. Clin Orthop Relat Res, 1997, 341: $250-256$

8 Kitaoka H B, Luo Z P, An K N. Reconstruction operations for acquired flatfoot: Biomechanical evaluation. Foot Ankle Int, 1998, 19:
203-207

9 Daly P J, Kitaoka H B, Chao E Y. Plantar fasciotomy for intractable plantar fasciitis: Clinical results and biomechanical evaluation. Foot Ankle, 1992, 13: 188-195

10 Berkelmans W A M, Poort H W, Slooff T J J H. A new method to analyse the mechanical behavior of skeletal parts. Acta Orth Scand, 1972, 34: 301-317

11 Cheng $\mathrm{H}$ Y, Lin C L, Chou S W, et al. Nonlinear finite element analysis of the plantar fascia due to the windlass mechanism. Foot Ankle Int, 2008, 29: 845-851

12 Arangio G A, Reinert K L, Salathe E P. A biomechanical model of the effect of subtalar arthroereisis on the adult flexible flat foot. Clin Biomech (Bristol, Avon), 2004, 19: 847-852

13 Gefen A. Stress analysis of the standing foot following surgical plantar fascia release. J Biomech, 2002, 35: 629-637

14 Camacho D L, Ledoux W R, Rohr E S, et al. A three-dimensional, anatomically detailed foot model: A foundation for a finite element simulation and means of quantifying foot-bone position. J Rehabil Res Dev, 2002, 39: 401-410

15 Jacob S, Patil M K. Three-dimensional foot modeling and analysis of stresses in normal and early stage Hansen's disease with muscle paralysis. J Rehabil Res Dev, 1999, 36: 252-263

16 Gefen A, Megido-Ravid M, Itzchak Y, et al. Biomechanical analysis of the three-dimensional foot structure during gait: A basic tool for clinical applications. J Biomech Eng, 2000, 122: 630-639

17 Chu T M, Reddy N P, Padovan J. Three-dimensional finite element stress analysis of the polypropylene, ankle-foot orthosis: Static analysis. Med Eng Phys, 1995, 17: 372-379

18 Patil K M, Braak L H, Huson A. Analysis of stresses in two-dimensional models of normal and neuropathic feet. Med Biol Eng Comput, 1996, 34: 280-284

19 Cheung J T, An K N, Zhang M. Consequences of partial and total plantar fascia release: A finite element study. Foot Ankle Int, 2006, 27: $125-132$

20 Tweed J L, Barnes M R, Allen M J, et al. Biomechanical consequences of total plantar fasciotomy: A review of the literature. J Am Podiatr Med Assoc, 2009, 99: 422-430

Open Access This article is distributed under the terms of the Creative Commons Attribution License which permits any use, distribution, and reproduction in any medium, provided the original author(s) and source are credited 